\title{
Current Treatment Approaches for Human Epidermal Growth Factor Receptor 2-Positive Breast Cancer in Adjuvant and Neoadjuvant Settings
}

\author{
Pankaj Goyal ${ }^{1}$ Dinesh Chandra Doval ${ }^{1}$ Chaturbhuj Agarwal ${ }^{1}$ Parveen Jain ${ }^{1}$ \\ Krushna Chaudhari ${ }^{1}$ Kshitij Domadia' Pallavi Redhu' ${ }^{1}$ Venkata Pradeep Babu Koyyala ${ }^{1}$ \\ Varun Goel ${ }^{1} \quad$ Ullas Batra ${ }^{1} \quad$ Vineet Talwar $^{1} \quad$ Sneha Bothra ${ }^{1}$
}

${ }^{1}$ Department of Medical Oncology, Rajiv Gandhi Cancer Institute and Research, New Delhi, India

Ind J Med Paediatr Oncol 2021;42:35-45.

\author{
Address for correspondence Chaturbhuj Agarwal, MD, DNB Medical \\ Oncology, Department of Medical Oncology, Rajiv Gandhi Cancer \\ Institute and Research, New Delhi 110085, India \\ (e-mail: chaturbhujagarwal06@ rediffmail.com).
}

\begin{abstract}
Keywords

- adjuvant

- human epidermal

growth factor

receptor 2 positive

breast cancer

- neoadjuvant

Breast cancer (BC) is the second most common cancer and the second leading cause of mortality among women globally. Approximately 20 to $25 \%$ of BC patients have amplification of the human epidermal growth factor receptor 2 (HER2) genes, a marker of poor prognosis. However, the introduction of anti-HER2-therapies (trastuzumab, followed closely by lapatinib, pertuzumab, trastuzumab emtansine, and neratinib) has changed the natural history of HER2-positive BC and improved the outcome in HER2-positive BC patients. The preeminence of anti-HER2 combination therapy in achieving complete inhibition of the various HER receptor dimers has been demonstrated in clinical studies. However, despite these therapeutic advances, tumors expressing estrogen receptor have poorer responses to targeted therapy and are more likely to relapse. A better understanding of resistance to existing anti-HER2 agents, along with the role played by the microenvironment and of interconnected signaling pathways, can permit tailor-made therapeutic options for each patient. This review aimed to evaluate treatment approaches for BC patients with HER2-positive disease in the adjuvant and neoadjuvant settings, also exploring the possibilities of extended duration of anti-HER2 maintenance therapy.
\end{abstract}

\section{Introduction}

Cancer was estimated to be the cause of 9.6 million deaths in 2018, making it the second leading cause of mortality. ${ }^{1}$ Breast cancer (BC) is the most frequently diagnosed cancer in women. Overall, it is the second leading cause of death in women. ${ }^{2}$ In 2018, there were over 2 million new cases of BC and over 627,000 women died due to this disease. ${ }^{3}$ In India, $\mathrm{BC}$ is ranked as the most common cancer among females, with the age-adjusted rate of $25.8 / 100,000$ women and mortality $12.7 / 100,000$ women. ${ }^{4}$

$\mathrm{BC}$ has evolved to be a heterogeneous disease clinically and pathologically. Amplification of the human epidermal growth factor receptor 2 (HER2) genes occurs in 20 to $25 \%$ of patients. ${ }^{5}$ Human epidermal growth factor receptor 2 is a transmembrane protein with tyrosine kinase activity encoded by the ERBB2 gene. The cell proliferation and tumor
DOI https://doi.org/ $10.1055 / \mathrm{s}-0041-1729726$ ISSN 0971-5851
(C) 2021. Indian Society of Medical and Paediatric Oncology This is an open access article published by Thieme under the terms of the Creative Commons Attribution-NonDerivative-NonCommercial-License, permitting copying and reproduction so long as the original work is given appropriate credit. Contents may not be used for commercial purposes, or adapted, remixed, transformed or built upon. (https://creativecommons.org/licenses/by-nc-nd/4.0/). Thieme Medical and Scientific Publishers Pvt. Ltd. A-12, 2nd Floor, Sector 2, Noida-201301 UP, India 
growth results from ligand-dependent and independent signaling through HER $2 .{ }^{6}$

HER2 overexpression is characterized by poorly differentiated high-grade tumors with reduced responses to traditional therapies and decreased survival. ${ }^{7,8}$

However, the effective advancement of HER2-directed therapies over the last two decades, initially in the palliative and then in the curative-intent settings, has dramatically improved the results in women affected by BC. ${ }^{9} \mathrm{~A}$ considerable number of HER2-targeted agents are available including monoclonal antibodies (MAbs), small-molecule inhibitors, and antibody-drug conjugates (ADC). ${ }^{10}$ The introduction of anti-HER2-therapies (trastuzumab, followed closely by lapatinib, pertuzumab, and trastuzumab emtansine) has changed the natural history of HER2-positive BC. Discovery of these therapies has resulted in a significant improvement in survival of both early and advanced settings. ${ }^{10-12}$

Currently, the standard of care in HER2-positive early $\mathrm{BC}$ includes neoadjuvant treatment with a combination of sequential chemo and HER2-targeted therapy, followed by breast surgery and radiotherapy (if indicated). ${ }^{13}$ This is ensued by 12 months of HER2-directed therapy, and depending on the tumor biology, endocrine adjuvant therapy. ${ }^{13}$ This article reviews the current treatment approaches for $\mathrm{BC}$ patients with HER2-positive disease in the adjuvant and neoadjuvant settings.

\section{The Function of Human Epidermal Growth Factor Receptors and Their Inhibitors}

The human epidermal growth factor (EGF), HER1, HER2, HER3, and HER4 are receptor tyrosine kinases involved in signal transduction pathways that modulate cellular processes. ${ }^{14} \mathrm{All}$ HER receptors excluding HER3 consist of an extracellular ligand-binding domain and intracellular tyrosine kinase domain $(\mathrm{T})$. The ligand (L) binds the receptor's extracellular domain, homodimerization, and heterodimerization, leading to tyrosine kinase domain phosphorylation and activation of downstream signaling pathways. ${ }^{14}$ Many novel HER2-targeting agents including the MAb trastuzumab, pertuzumab, and ADC trastuzumab emtansine (T-DM1), and the tyrosine kinase inhibitors (TKIs) like lapatinib and neratinib, have been developed; these agents target the signal transduction pathway downstream from HER2 ( - Fig. 1 and - Table 1). ${ }^{8,15,16}$

Human Epidermal Growth Factor Receptor 2 Inhibitors Trastuzumab is a humanized MAb. It is the first HER2-targeting drug introduced to BC clinics and remains a major component of the most effective regimens used to treat HER2-positive BC. Pertuzumab is an alternative monoclonal anti-HER2 antibody, which binds HER2 at a different location than trastuzumab. It inhibits the formation of HER2-HER3 heterodimers. ${ }^{15}$ Lapatinib is a reversible small-molecule TKIs, which has activity against the tyrosine kinase ATP-binding pocket of HER1 as well as HER2. ${ }^{8}$

Trastuzumab emtansine is an ADC. This new drug enables targeted delivery of cytotoxic molecules to the tumor, thereby increasing efficiency and simultaneously reducing toxicity. ${ }^{15}$

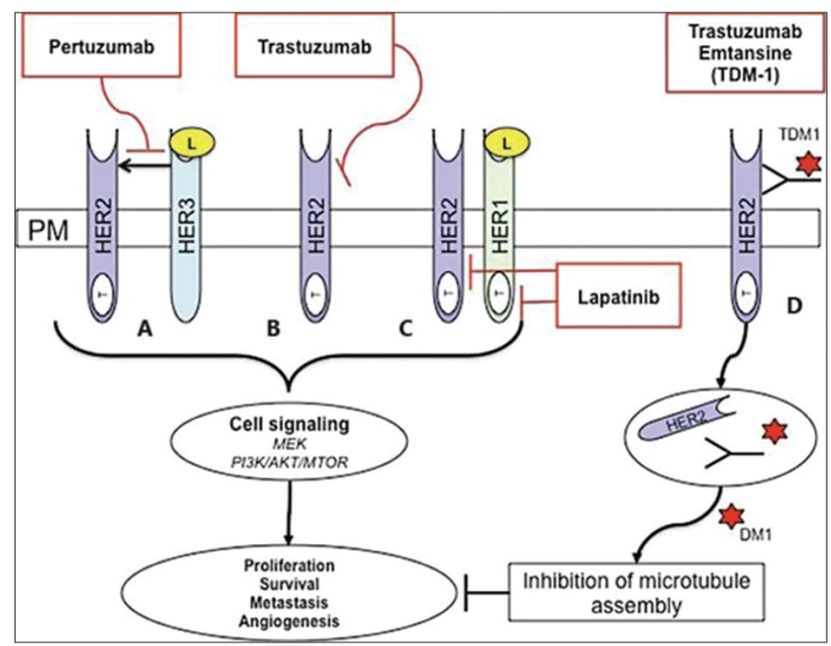

Fig. 1 Human epidermal growth factor receptor 2 (HER2) targeted therapies for the treatment of metastatic breast cancer. ${ }^{14}$

Table 1 Antihuman epidermal growth factor receptor 2 blocking agents and mechanisms of action ${ }^{16}$

\begin{tabular}{|l|l|l|}
\hline $\begin{array}{l}\text { Class of } \\
\text { compounds }\end{array}$ & Agent (s) & Mechanism of action \\
\hline $\begin{array}{l}\text { Monoclonal } \\
\text { antibody }\end{array}$ & Trastuzumab & $\begin{array}{l}\text { Prevent formation of HER2- } \\
\text { containing heterodimers, anti- } \\
\text { body-dependent cell-mediated } \\
\text { cytotoxicity (ADCC), disruption of } \\
\text { downstream signaling pathways, } \\
\text { inhibition of cleavage of HER2, } \\
\text { and promoting endocytosis of } \\
\text { HER2 receptor }\end{array}$ \\
\hline $\begin{array}{l}\text { Antibody } \\
\text { drug } \\
\text { conjugates }\end{array}$ & Pertuzumab & $\begin{array}{l}\text { It exerts its function by binding } \\
\text { with an epitope on the extra- } \\
\text { cellular domain of HER2 recep- } \\
\text { tors, therefore preventing the } \\
\text { formation of HER2-containing } \\
\text { heterodimers }\end{array}$ \\
\hline $\begin{array}{l}\text { Small- } \\
\text { molecule } \\
\text { inhibitors }\end{array}$ & Lapatinib & $\begin{array}{l}\text { Trastuzumab's action Selective } \\
\text { delivery of the antimicrotubule } \\
\text { agent DM1 }\end{array}$ \\
\hline $\begin{array}{l}\text { Reversible and dual small } \\
\text { molecular inhibitor of HER2 and } \\
\text { HER1 with prolonged inhibition } \\
\text { of tyrosine phosphorylation in } \\
\text { tumor cells }\end{array}$ \\
\hline $\begin{array}{l}\text { Irreversible dual inhibitor of EGFR } \\
\text { and HER2 }\end{array}$ \\
\hline Afatinib, \\
neratinib
\end{tabular}

Abbreviations: EGFR, epidermal growth factor receptor; HER2, human epidermal growth factor receptor 2; T-DM1, trastuzumab emtansine.

\section{Human Epidermal Growth Factor Receptor 2 Inhibitors and Tumor Resistance}

While BC will be cured in some patients treated in the adjuvant setting, a fraction is predicted to eventually recur. Tumors harbor de novo or acquire resistance to therapeutic inhibitors of HER2. ${ }^{17,18}$ General mechanisms of resistance to HER2-targeted therapies transpire at three levels. The first involves mechanisms inherent in the target, such as molecular changes in the target receptor; the p95HER2 expression, which is a truncated HER2 receptor; and HER2 gene amplification. ${ }^{19}$ Second, resistance involving parallel signaling 
pathways bypassing HER2 inhibition, like amplified activation of HER3, unusual activation of pathways downstream of the receptor, and compensatory crosstalk with other pathways, can also occur. ${ }^{19}$ The third mechanism involves resistance due to defects in the apoptosis pathway in tumor cells or extrinsic host factors contributing to the action of the drugs. ${ }^{19}$

\section{Biological Insights into Improved Human Epidermal Growth Factor Receptor 2 Targeting}

\section{Inhibition of Human Epidermal Growth Factor Receptor Family Dimerization}

The dimerization of HER family members is a known mechanism of resistance to anti-HER2 therapy. ${ }^{20}$ All members of the HER family can form dimers together, but HER2 and HER3 form a predominantly potent heterodimer, which is important in BC growth and development. Although HER3 does not exert tyrosine kinase activity on its own, HER2 dimerization considerably increases downstream signaling activity ${ }^{21,22}$ and provides an escape mechanism for HER2 inhibition. ${ }^{23,24}$ Pertuzumab is a humanized MAb bound to the HER2 dimerization domain. It is an inhibitor of HER2/HER3 dimerization and also induces antibody-dependent cellular cytotoxicity. Although pertuzumab has some clinical activity of its own when used in combination with trastuzumab, the dual binding to HER2 results in synergistic action. ${ }^{8}$

In the pertuzumab and trastuzumab (CLEOPATRA) phase III clinical evaluation trial, 808 patients with metastatic BC (MBC) who had not previously received anti-HER2 therapy for metastatic disease were treated with the combination of pertuzumab and trastuzumab with docetaxel. Median progression-free survival as investigators assessed, increased by 6 months (hazard ratio [HR]: $0.65, p<0.001$ ), and the objective response rate improved by $10.8 \% .{ }^{25}$ The combination also leads to a marked increase in overall survival (OS) by almost 16 months compared with standard trastuzumab plus docetaxel (HR: $0.68, p<0.001){ }^{26}$ Since this research, pertuzumab plus trastuzumab has replaced trastuzumab as the standard of care for first-line metastatic BC.

Many TKIs such as lapatinib and neratinib have recently been developed to target the downstream signal transduction pathway from HER2. It is effective against HER2 amplification in early as well as metastatic $\mathrm{BC}$ when given in combination with trastuzumab..$^{27,28}$ However, most HER2 somatic mutations were resistant to lapatinib in preclinical studies. ${ }^{29}$ Neratinib has emerged as a potent inhibitor of HER2 activity as yet another HER2-targeting TKI. It is an irreversible pan-inhibitor of HER2 and HER1/EGFR and has been found to be more effective in blocking HER2 activation than lapatinib. ${ }^{30,31}$

\section{Optimizing Human Epidermal Growth Factor Receptor 2-Targeted Therapy for Breast Cancer}

The advancement of HER2-targeted therapies has revolutionized women's treatment of HER2-positive BC. These targeted therapies have improved their outcomes signifi-

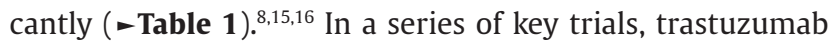
was tested in the adjuvant setting, with the success of anti-HER2 therapy in (MBC). Major international trials were the Herceptin Adjuvant (HERA) Trial study, the National Surgical Adjuvant Breast and the Bowel Project (NSABP) B-31 trial, the BC International Research Group 006 trial, and the North Central Cancer Treatment Group N9831 of more than 13,000 women with HER-2-positive early BC.

Patients treated with trastuzumab in the HERA trial experienced a $46 \%$ lower risk of a first event (HR: 0.54 ; $95 \%$ confidence interval $[\mathrm{CI}]: 0.43-0.67 ; p<0.0001$ ) than patients under observation, at 1 -year median follow-up. This corresponded to an absolute disease-free survival (DFS) benefit favoring trastuzumab of $8.4 \%$ at 2 years. ${ }^{32}$

\section{Present Standards of Care in Human Epidermal Growth Factor Receptor 2-Positive Early Breast Cancer}

For HER2-positive early BC, the current standard of care includes trastuzumab (with or without pertuzumab) and chemotherapy, ${ }^{8}$ established on the basis of benefit of trastuzumab over standard care in both the adjuvant and neoadjuvant settings, shown in several clinical studies. ${ }^{8,32-35}$ In the phase III HERA trial, 1 year of adjuvant trastuzumab was associated with significant improvements in both 10-year rates of DFS (69 vs. 63\%; HR: 0.76) and 12-year rates of OS (79\% vs. 73\%; HR: 0.74 ) compared with observation. ${ }^{8,32,33}$

In the phase III NeoAdjuvant Herceptin (NOAH) trial, HER2-positive patients treated with trastuzumab plus chemotherapy had a significantly higher rate of pathologic complete response ( $\mathrm{pCR}$ ) compared with patients who received chemotherapy only (38.5 vs. $19.5 \%$; HR: $0.29, p=0.0135$ ) and these patients remained disease-free longer (5-year event-free survival [EFS] 58 vs. $43 \%$; HR: $0.64, p=0.016) .{ }^{35}$ Even though trastuzumab has demonstrated efficacy in early BC, a significant proportion of patients in due course of time will progress. ${ }^{8}$ In the HERA trial, after 10 years of follow-up, 28.8\% of patients treated with trastuzumab experienced disease progression. ${ }^{8,32,33}$ Likewise, in the NOAH trial, at only 5 years posttreatment with trastuzumab, $42 \%$ of patients treated experienced a disease recurrence. ${ }^{35}$ This establishes that for several patients with HER2-positive BC, there is still a significant unmet need, and research in recent years has focused on identifying novel approaches to adjuvant and neoadjuvant therapy that can improve outcomes for these patients. ${ }^{8}$

\section{Human Epidermal Growth Factor Receptor 2-Targeted Adjuvant Therapy Other Approaches}

Many novel HER2-targeting agents have been studied including dual HER2 blockade. These agents have the potential for being used as adjuvant therapy for patients with nonmetastatic HER2-positive BC.

\section{Lapatinib}

Lapatinib has been evaluated as prospective adjuvant therapy for HER2-positive BC, either alone or in combination with 
trastuzumab. In the Tykerb Evaluation after Chemotherapy trial, lapatinib was compared with placebo in women with HER2-positive early BC, who had previously received adjuvant chemotherapy but not trastuzumab. ${ }^{36}$ The study results showed that single-agent lapatinib failed to demonstrate a significant DFS benefit over placebo (HR: 0.83, 95\% CI: 0.701.00). However, exploratory analyses showed a marginal benefit for patients with HER2-positive disease confirmed by central fluorescence in situ hybridization. ${ }^{36}$ Patients who received single-agent lapatinib had poorer outcomes relative to patients who received trastuzumab in the adjuvant lapatinib and/or trastuzumab treatment optimization (ALTTO) trial (HR: 1.34). ${ }^{37}$ Lapatinib and trastuzumab combination appeared to improve outcomes, as patients who received both lapatinib and trastuzumab, either in combination $(\mathrm{L}+\mathrm{T})$ or in sequence $(\mathrm{T} \rightarrow \mathrm{L})$, showed significant DFS rates $(\mathrm{L}+\mathrm{T}: 88 \%$ and $\mathrm{T} \rightarrow \mathrm{L}: 87 \%)$ as compared with patients who received single agent (lapatinib L: $82 \%$ ). However, at the 5 years of follow-up, the combination did not sufficiently improve either DFS or OS compared with single-agent trastuzumab. ${ }^{38} \mathrm{At}$ present, lapatinib is not being further studied, either alone or in combination with trastuzumab, in the adjuvant setting.

\section{Neratinib}

Neratinib studies had been successful in the adjuvant setting. A multicenter, randomized, double-blind, placebo-controlled, phase III study, neratinib after trastuzumab-based adjuvant therapy in HER2-positive BC (ExteNET) compared neratinib (240 mg daily) to placebo. ${ }^{39}$ This study was conducted at 495 centers across America, Europe, Asia, Australia, and New Zealand. The women included in this study were with stage I to III HER2-positive BC, who had completed neoadjuvant and adjuvant trastuzumab for up to 2 years (amended to 1 year) before randomization. Included patients were stage I to III node positive and node negative with tumors $>1 \mathrm{~cm}$ in size. In this study, neratinib improved invasive DFS (iDFS) by 2 years by $2.3 \%$ over placebo (HR: $0.67, p=0.009$ ). ${ }^{39}$

This improvement was most discrete in patients with hormone receptor (HR) -positive tumors (HR: 0.51, $p=0.001$ ). This study suggested that neratinib may provide a treatment option for this subset of patients who have a long-term constant risk of relapse on trastuzumab.

Furthermore, at 5-year follow-up, the benefit with neratinib was maintained with a $2.5 \%$ absolute improvement in iDFS (90.2 vs. 87.7\%; HR: 0.73, $p=0.008$ ) for the whole population. ${ }^{40}$ This improvement in iDFS was most apparent in HR-positive patients (91.2 vs. 86.8\%; HR: 0.60, $p=0.002) .{ }^{40} \mathrm{It}$ was observed that patients who have completed adjuvant trastuzumab $<1$ year prior to the start of the study showed the maximum benefit with neratinib. ${ }^{40}$

\section{Pertuzumab}

APHINITY study examined the pertuzumab (P) and trastuzumab ( $\mathrm{T}$ ) combination as adjuvant treatment, compared with standard trastuzumab plus chemotherapy $(\mathrm{C})$ in 4805 patients with HER2-positive early BC. ${ }^{41}$ The study included patients who had either node-positive disease or node-negative disease ( $\mathrm{pNO}$ ) and a tumor size of $>1.0 \mathrm{~cm}$.
Eligible patients were with pN0, T1b tumors with high-risk features. A total of 4805 patients were randomized to $\mathrm{C}$ and $\mathrm{T}$ plus either $P(n=2400)$ or placebo $(n=2405){ }^{41}$

This study reached its primary end point of improved iDFS at 3 years ( 94.1 vs. 93.2\%; HR: 0.81, $p=0.045$ ), the gain magnitude was small, and there was no related change in OS (97.7\% for both groups; HR: 0.89, $p=0.467$ ).

Analysis in the subgroup suggested a somewhat greater effect on ER-negative tumors (HR: 0.76, $p=0.085$ ). Treatment with pertuzumab was associated with an increase in grade $\geq 3$ diarrhea (9.8 vs. $3.7 \%$ ). ${ }^{41}$ Results from key phase III trials are summarized in - Table 2.

\section{Ado-trastuzumab Emtansine}

T-DM1 is an immunoconjugate of trastuzumab with a microtubule inhibitor. It is a derivative of fungal toxin emtansine (DM1), which has three capabilities: anti-HER2 function of trastuzumab, DM1-induced cytotoxicity, and tissue-specific expression. ${ }^{42}$

The KATHERINE trial compared adjuvant therapy with T-DM1 to adjuvant trastuzumab in patients with HER2-positive early BC who had residual disease after neoadjuvant therapy involving a minimum of six cycles of taxane-based chemotherapy and 9-week trastuzumab. ${ }^{42}$ Patients randomized received either 14 cycles of ado-trastuzumab emtansine or 14 cycles of trastuzumab. Treatment with 14 cycles of adjuvant T-DM1 resulted in a significant improvement in the rates of iDFS compared with adjuvant trastuzumab. ${ }^{43}$

iDFS favored T-DM1 in all prespecified patient subgroups. At 3 years, a larger proportion of patients receiving T-DM1 remained free of distant recurrence (89.7 vs. 83.0\%; HR: 0.60). There was a trend toward improved OS in patients receiving T-DM1 (although the OS data are not yet mature). This study concluded that adjuvant T-DM1 in patients with invasive residual disease demonstrated a statistically significant benefit with clinically meaningful improvements in iDFS compared with trastuzumab, and tailoring therapy based on pathological response improves outcomes. ${ }^{43}$

\section{Neoadjuvant Therapy for Human Epidermal Growth Factor Receptor 2-Positive Breast Cancer}

Trastuzumab as neoadjuvant therapy provides significant clinical benefits and decreases the rate of distant metastasis. ${ }^{44}$ Clinical studies have shown that trastuzumab-based neoadjuvant therapy has a higher rate of PCR (defined as the absence of residual cancer in the breast or axillary lymph node pathology) in the treatment of HER2-positive BC. ${ }^{45}$

At first, a small randomized trial was conducted to determine whether the addition of trastuzumab to chemotherapy in the neoadjuvant setting could increase $(\mathrm{pCR})$ rate in patients with HER2-positive disease. This study confirmed the role of trastuzumab in the neoadjuvant scenario. ${ }^{46}$ Another multicenter, open-label, randomized phase III study was NOAH trial. ${ }^{47}$ A sustained improvement in the EFS rate with neoadjuvant therapy involving trastuzumab was achieved after a 5.4-year follow-up (58 vs. 43\%; HR: 0.64). A strong association with $\mathrm{pCR}$ was revealed in patients given trastuzumab. 
Table 2 Disease-free survival and overall survival rates for phase III adjuvant trials of novel human epidermal growth factor receptor 2 inhibitors ${ }^{8}$

\begin{tabular}{|c|c|c|c|c|c|c|}
\hline Agent/trial & Disease setting & Regimen & $n$ & Follow-up & DFS & OS \\
\hline Lapatinib & & & & & & \\
\hline $\begin{array}{l}\text { ALTTO trial } \\
\text { Piccart-Gebhart } \\
\text { et al }{ }^{37}\end{array}$ & $\begin{array}{l}\text { Stage I-III adjuvant } \\
\text { therapy }\end{array}$ & $\begin{array}{l}\text { Lapatinib }+ \\
\text { trastuzumab } \\
\text { Lapatinib }(34 \mathrm{w}) \rightarrow \\
\text { Trastuzumab }(12 \mathrm{w})\end{array}$ & 8,381 & $6 y$ & $\begin{array}{l}\text { 85\% (HR: 0.86) } \\
84 \% \text { (HR: } 0.93)\end{array}$ & $\begin{array}{l}\text { 93\% (HR: 0.86) } \\
\text { 92\% (HR: } 0.88)\end{array}$ \\
\hline $\begin{array}{l}\text { Moreno-Aspitia } \\
\text { et } \mathrm{al}^{38}\end{array}$ & & $\begin{array}{l}\text { Trastuzumab } \\
\text { Lapatinib }\end{array}$ & & & $\begin{array}{l}82 \% \\
82 \%(H R: 1.34)\end{array}$ & $\begin{array}{l}91 \% \\
93 \% \text { (HR: } 1.36)\end{array}$ \\
\hline $\begin{array}{l}\text { TEACH-Goss } \\
\text { et al }{ }^{36} \\
\text { Neratinib }\end{array}$ & $\begin{array}{l}\text { Stage I-III delayed } \\
\text { adjuvant therapy. Prior } \\
\text { trastuzumab unless } \\
\text { contraindicated }\end{array}$ & $\begin{array}{l}\text { Lapatinib } \\
\text { Placebo }\end{array}$ & 3,147 & $\begin{array}{l}47.4 \mathrm{mo} \\
48.3 \mathrm{mo}\end{array}$ & $\begin{array}{l}87 \% \text { (HR: 0.83) } \\
83 \%\end{array}$ & $\begin{array}{l}\text { 94\% (HR: 0.99) } \\
94 \%\end{array}$ \\
\hline $\begin{array}{l}\text { ExteNET-Martin } \\
\text { et al }{ }^{40} \\
\text { Pertuzumab }\end{array}$ & $\begin{array}{l}\text { Stage I-III delayed } \\
\text { adjuvant therapy. Prior } \\
\text { trastuzumab }\end{array}$ & $\begin{array}{l}\text { Neratinib } \\
\text { Placebo }\end{array}$ & 2,840 & $5 y$ & $\begin{array}{l}90.2 \% \text { (HR: } 0.73 \text { ) } \\
87.7 \% *\end{array}$ & Not mature \\
\hline $\begin{array}{l}\text { APHINITY-von } \\
\text { Minckwitz et al }{ }^{41}\end{array}$ & $\begin{array}{l}\text { Stage II-III adjuvant } \\
\text { therapy }\end{array}$ & $\begin{array}{l}\text { Pertuzumab } \\
\text { (plus chemo and } \\
\text { trastuzumab) } \\
\text { Placebo (plus chemo } \\
\text { and trastuzumab) }\end{array}$ & 4,805 & $36 \mathrm{mo}$ & $\begin{array}{l}94.1 \%(\mathrm{HR}: \\
0.81)^{*} \\
93.2 \%{ }^{*}\end{array}$ & $\begin{array}{l}97.7 \%(\mathrm{HR}: \\
0.89) \\
97.7 \%\end{array}$ \\
\hline
\end{tabular}

Abbreviations: ALTTO, adjuvant lapatinib and/or trastuzumab treatment optimization; Chemo, chemotherapy; DFS, disease-free survival; ET, endocrine therapy; HR, hazard ratio; OS, overall survival.

pCR improved from 22 to $43 \%(p<0.001){ }^{35}$ In the trial of Taxol Epirubicin Cyclophosphamide Herceptin Neoadjuvant (TECHNO), 217 HER2-positive patients obtained four cycles of EC (epirubicin and cyclophosphamide) followed by four cycles of $\mathrm{TH}$ (paclitaxel and trastuzumab) as neoadjuvant therapy. pCR was obtained in nearly $38.7 \%$. Three-year DFS ( 88 vs. $71 \% ; p=0.003$ ) and OS (96 vs. $85 \% ; p=0.007$ ) were improved. ${ }^{48}$

Other trials, such as the American Z1041 trial, ${ }^{49}$ Gepar Quattro study, ${ }^{50}$ and HannaH trial, ${ }^{51}$ also enrolled HER2-positive BC patients with comparable inclusion criteria as TECHNO. These trials also evaluated treatment with chemotherapy plus trastuzumab as concurrently or consequence regimens (-Table $\mathbf{3}$ ).

\section{Other Therapy in the Neoadjuvant Setting}

The GeparQuinto ${ }^{52}$ phase III trial compared the efficacy of two HER2-targeted drugs, lapatinib and trastuzumab, with the combination of four cycles of chemotherapy with EC, followed by docetaxel. The results of the study indicated that the arm of trastuzumab showed $\sim 7 \%$ more pCR than the arm of lapatinib (30.3 vs. $22.7 \%$; $p=0.04$ )..$^{52}$

The lapatinib with trastuzumab for HER2-positive early BC (NeoALTTO) trial, ${ }^{27}$ an international, randomized, open-label, multicenter, phase III study, compared the efficacy of lapatinib or trastuzumab monotherapy, or the concomitant lapatinib and trastuzumab regimen, in addition to paclitaxel, in the neoadjuvant setting. In the combination arm, noticeable progress on pCR of 51\% was observed, which is almost twice as much as the other two monotherapies against HER2 (29.5\% in trastuzumab alone and 24.7\% in lapatinib alone, $p<0.001) .{ }^{27}$ Other studies with the dual inhibitory regimen, NSABP B-41 study, ${ }^{53}$ NeoSphere trial, ${ }^{54,55}$ and the TRYPHAENA trial ${ }^{56}$ details are illustrated in - Table 3.

\section{Novel Strategies to Overcome Resistance to Human Epidermal Growth Factor Receptor 2-Targeted Therapy}

Replacement of Current Antihuman Epidermal Growth Factor Receptor 2 Therapies for Improved Antihuman Epidermal Growth Factor Receptor 2 Drugs Antibody-Drug Conjugates

ADCs provide a wider therapeutic window by providing more effective and specific drug delivery. ADCs leverage target selectivity of MAbs to deliver cytotoxic drugs to antigen-expressing cells to enhance tumor selectivity and reduce damage to normal cells. ${ }^{57}$ Several anti-HER2 ADCs in clinical development are listed in - Table 4.

\section{Novel Tyrosine Kinase Inhibitors}

There are several novel TKIs in clinical development listed in - Table 5. 
Table 3 Optimal clinical trials in the neoadjuvant setting for human epidermal growth factor receptor 2-positive breast cancer ${ }^{57}$

\begin{tabular}{|c|c|c|c|c|c|}
\hline $\begin{array}{l}\text { Drug or study } \\
\text { name }\end{array}$ & $\begin{array}{l}\text { Neoadjuvant } \\
\text { chemotherapy }\end{array}$ & $\begin{array}{l}\text { Number of } \\
\text { patients }\end{array}$ & $\begin{array}{l}\text { Pathological } \\
\text { complete response } \\
\text { (\%) }\end{array}$ & Comments & References \\
\hline $\begin{array}{l}\text { The NSABP } \\
\text { B-41 trial }\end{array}$ & $\mathrm{AC} \rightarrow \mathrm{TH}$ or TL or THL & $\begin{array}{l}181 \text { versus } \\
174 \text { versus } \\
174\end{array}$ & $\begin{array}{l}52.5 \% \text { versus } 53.2 \% \\
\text { versus } 62.0 \%\end{array}$ & $\begin{array}{l}\mathrm{H}+\mathrm{L} \text { no better. All } \\
\text { patients received } \\
\text { anthracyclines }\end{array}$ & Robidoux et $a^{53}$ \\
\hline Pertuzumab & Do + H versus Do + & 107 versus & $29.0 \%$ versus $45.8 \%$ & $\begin{array}{l}\geq 0.6 \text {. Combination } P+ \\
H \text { results in better } P C R\end{array}$ & Gianni et a $\left.\right|^{54,55}$ \\
\hline The NeoSphere trial & $\begin{array}{l}P+H \text { versus Do }+P \\
\text { versus } P+H\end{array}$ & $\begin{array}{l}\text { versus } 107 \\
\text { versus } 96\end{array}$ & $\begin{array}{l}\text { versus } 24.0 \% \text { versus } \\
16.8 \%\end{array}$ & & \\
\hline The TRYPHAENA trial & $\begin{array}{l}\mathrm{FEC}+\mathrm{HP} \rightarrow \mathrm{Do}+\mathrm{HP} \\
\text { versus } \mathrm{FEC} \rightarrow \mathrm{Do}+\mathrm{HP} \\
\text { versus TCHP }\end{array}$ & $\begin{array}{l}223 \text { patients } \\
\text { in total }\end{array}$ & $\begin{array}{l}62 \% \text { versus } 57 \% \text { versus } \\
66 \%\end{array}$ & $\begin{array}{l}\mathrm{TCH}+\mathrm{P} \text { is an active } \\
\text { combination }\end{array}$ & Schneeweiss et al ${ }^{56}$ \\
\hline
\end{tabular}

Abbreviations: A, Adriamycin; BC, breast cancer; C, cyclophosphamide; Do, docetaxel; E, epirubicin; F, 5FU; H, trastuzumab; human epidermal growth factor receptor 2; L, lapatinib; M, methotrexate; P, pertuzumab; pCR, pathologic complete response; T, paclitaxel; TC, docetaxel plus carboplatin; T-DM1, trastuzumab emtansine.

Goyal et al: Current treatment approaches for HER2+ BC.

Table 4 Human epidermal growth factor receptor 2-directed antibody-drug conjugates in clinical development

\begin{tabular}{|c|c|c|c|c|c|c|}
\hline Agent & $\begin{array}{l}\text { Anti-HER2 MAb/payload } \\
\text { (target) }\end{array}$ & $\begin{array}{l}\text { Drug to } \\
\text { antibody } \\
\text { ratio }\end{array}$ & Linker drug & $\begin{array}{l}\text { Phase of } \\
\text { development }\end{array}$ & $\begin{array}{l}\text { ORR in } \\
\text { HER2- } \\
\text { positive }\end{array}$ & $\begin{array}{l}\text { ORR in HER2 low } \\
(\mathrm{IHC} 1+/ 2+/ \mathrm{IS}\end{array}$ \\
\hline T-DM158 & T-DM1 (antitubulin) & 3.5 & Noncleavable & US FDA approved & $43.6 \%$ & - \\
\hline $\begin{array}{l}\text { Trastuzumab } \\
\text { deruxtecan } \\
\text { (DS-8201a) }{ }^{59}\end{array}$ & $\begin{array}{l}\text { Trastuzumab/exatecan } \\
\text { derivative } \\
\text { (topoisomerase I inhibitor) }\end{array}$ & 8 & Cleavable & $\begin{array}{l}\text { II/III } \\
\text { NCT03248492 } \\
\text { NCT03529110 } \\
\text { NCT03523585 }\end{array}$ & $54.5 \%$ & $50 \%$ \\
\hline SYD98560 & $\begin{array}{l}\text { Duocarmycin derivative } \\
\text { (alkylating agent) }\end{array}$ & 2.8 & Cleavable & $\begin{array}{l}\text { III } \\
\text { NCT03262935 }\end{array}$ & $33 \%$ & $\begin{array}{l}\mathrm{HR}^{+} 27 \% \\
\mathrm{HR}^{-} 40 \%\end{array}$ \\
\hline XMT-1522 & $\begin{array}{l}\text { XMT-1519/monomethyl } \\
\text { auristatin (antitubulin) }\end{array}$ & 12 & Cleavable & $\begin{array}{l}\text { I } \\
\text { NCT02952729 }\end{array}$ & Unknown & Unknown \\
\hline
\end{tabular}

Abbreviations: $\mathrm{ADC}$, antibody-drug conjugate; $\mathrm{HR}^{-}$, hormone receptor-negative; $\mathrm{HR}^{+}$, hormone receptor-positive; IHC, immunohistochemistry; MAb, monoclonal antibody; NCT, ClinicalTrials.gov identifier; ORR, overall response rate; T-DM1, trastuzumab emtansine; US FDA, United States Food and Drug Administration.

\section{Escalating or De-escalating Adjuvant Therapy in Human Epidermal Growth Factor Receptor 2-Positive Breast Cancer}

Recently, many studies have investigated modifications of the schedule of treatment with trastuzumab by either making it shorter and less toxic (de-escalation) or more effective with dual HER2 inhibition or extended treatment duration (escalation). ${ }^{63}$

Seven randomized trials investigated whether a shorter regimen of adjuvant trastuzumab may be as effective as 1-year of trastuzumab, but with fewer side effects. In four trials, trastuzumab was given in combination with chemotherapy in the experimental arm with the objective to investigate drug synergism (FinHer, E2198, SOLD, and Short-HER trials), and three trials compared 6- to the 12-month duration of trastuzumab (the Hellenic trial, PHARE, and PERSEPHONE).

The FinHer study randomized ${ }^{64} 1,010$ women with axillary node-positive or high-risk node-negative $\mathrm{BC}$ to obtain three cycles of docetaxel or vinorelbine, followed by three cycles of fluorouracil (F), epirubicin (E), and cyclophosphamide $(C)$ in both groups. Two-hundred thirty-two patients with HER2-positive BC were further treated with trastuzumab or no additional therapy. Even though trastuzumab was given for a shorter duration, distant DFS (83.3 vs. 73\%) and OS (91.3 vs. $82.3 \%$ ) favored the trastuzumab arm after a median of 8 years. ${ }^{64}$

In the SOLD trial,65 2,176 patients with early-stage HER2-positive BC were randomized (1:1) to the 9-week trastuzumab arm or the 12-month trastuzumab arm. Both arms received three cycles of docetaxel $\left(80 \mathrm{mg} / \mathrm{m}^{2}\right.$ or $\left.100 \mathrm{mg} / \mathrm{m}^{2}\right)$ and trastuzumab (q3 weekly), followed by three cycles of chemotherapy. ${ }^{65}$ The 9-week arm received no further treatment, while those in the 12-month arm received additional 14 cycles of trastuzumab every 3 weeks. The trial failed to establish that 9 weeks of adjuvant trastuzumab was noninferior to the standard 12 months in terms of DFS. ${ }^{65}$ The 
Table 5 New antihuman epidermal growth factor receptor 2 agents and combinations ${ }^{62}$

\begin{tabular}{|c|c|}
\hline & Novel HER2 antibodies \\
\hline Agent & Mechanism of action \\
\hline Margetuximab (MGAH22) & $\begin{array}{l}\text { Optimized Fc domain for enhanced binding to the activating low-affinity Fc receptor, } \\
\text { FcyRIIIA }\end{array}$ \\
\hline MCLA-128 & $\begin{array}{l}\text { IgG1 bispecific antibody with enhanced ADCC activity targeting both HER2 and HER3 } \\
\text { receptors }\end{array}$ \\
\hline ZW-25 & The bispecific antibody directed against two distinct epitopes of HER2 \\
\hline \multicolumn{2}{|l|}{$A D C$} \\
\hline SYD 985 & Trastuzumab with an alkylant prodrug DUBA (duocarmycin derivate) payload \\
\hline DS-8201 & HER2 antibody attached to topoisomerase I inhibitor (DXd) payload \\
\hline \multicolumn{2}{|l|}{ TKIs } \\
\hline Neratinib & Oral TKI that irreversibly inhibits HER1, HER2 and HER 4 \\
\hline Tucatinib & Oral TKI, ATP competitive, selectively inhibits HER2 relative to EGFR \\
\hline Poziotinib & Irreversible oral TKI, pan-HER kinase inhibitor \\
\hline Pyrotinib & Irreversible oral, TKI pan-HER kinase inhibitor \\
\hline \multicolumn{2}{|l|}{ Immune approaches } \\
\hline Atezolizumab & Anti-PD-L1 antibody \\
\hline Pembrolizumab & Anti-PD-L1 antibody \\
\hline \multicolumn{2}{|l|}{ CDK4/6 inhibitor } \\
\hline Palbociclib & CDK4/6 inhibitor \\
\hline Abemaciclib & CDK4/6 inhibitor \\
\hline Ribociclib & CDK4/6 inhibitor \\
\hline \multicolumn{2}{|l|}{ PI3K inhibitors } \\
\hline Alpelisib & $\alpha$-specific PI3K inhibitor \\
\hline Copanlisib & Pan-class PI3K inhibitor \\
\hline Taselisib & $\beta$-sparring PI3K inhibitor \\
\hline
\end{tabular}

Abbreviations: ADC, antibody-drug conjugates; ADCC, antibody-dependent cell-mediated cytotoxicity; CBR, clinical benefit rate; CDK, cyclin-dependent kinase; DCR, disease control rate; HER2, human epidermal growth factor receptor 2; NR, not reported; ORR, overall response rate; PI3K, phosphatidylinositol-3-kinase; T, trastuzumab; TKI, tyrosine kinase inhibitor.

shorter trastuzumab treatment was safer to the heart than the longer treatment. In the 9-week group, there were 22 protocol-defined cardiac adverse events compared with 42 in patients receiving 1 year of trastuzumab $(p=0.012) .{ }^{65}$

In the short-HER study, ${ }^{66,67}$ patients were randomly selected to receive 1 year of trastuzumab plus chemotherapy ("long" group) or 9 weeks of trastuzumab plus chemotherapy ("short" group). The primary end points were DFS and $\mathrm{OS}$. The secondary end points included failure rate at 2 years and the incidence of cardiac events. ${ }^{66,67}$ The 5-year DFS was not noninferior ( 87.5 vs. $85.4 \%$ in the long and short groups, respectively, HR: $1.15,90 \% \mathrm{CI}$ : $[0.91,1.46])$. In an analysis of DFS in patients with the earlier-stage disease (stage I and II) as compared with those with locally advanced disease (stage III), the shorter duration was not inferior to the longer one. There was no difference in OS at 5 years. ${ }^{66}$

PERSEPHONE was a noninferiority trial, which randomized patients of early BC to 6 months of trastuzumab versus the standard 12 months in 4,088 patients between 2007 and
2015. ${ }^{68,69}$ Patients were randomized to receive either 6-month or 12-month trastuzumab delivered every 3 weeks intravenously (loading dose of $8 \mathrm{mg} / \mathrm{kg}$ followed by maintenance doses of $6 \mathrm{mg} / \mathrm{kg}$ ) or subcutaneously (600 mg), given in combination with chemotherapy (concurrently or sequentially). The margin of noninferiority was 3\% difference in DFS of the 6-month arm versus 12-month arm. Randomization was done before the tenth cycle of trastuzumab. The researchers found nearly identical results between the two treatment arms at a mean follow-up of 5 years: DFS was $89.4 \%$ for women in the 6-month arm and $89.8 \%$ for women in the 12 -month arm indicating a noninferiority of 6-month trastuzumab in women with carefully selected early BC and adding an option of 6-month trastuzumab in resource-limited setting. . $^{68,69}$

\section{Seven-Year Follow-Up of Adjuvant Paclitaxel and Trastuzumab Trial}

This study included 410 HER2-positive BC patients with tumors $3 \mathrm{~cm}$ or smaller and negative nodes. They received 
12 weeks of paclitaxel $\left(80 \mathrm{mg} / \mathrm{m}^{2}\right)$ with trastuzumab followed by 9 months of trastuzumab. ${ }^{70}$ The main end point was DFS. It also analyzed recurrence-free interval, BC-specific survival, and OS.

After a median follow-up of 4 years, the first study from the Adjuvant Paclitaxel and Trastuzumab trials revealed an intrusive DFS of 98.7 over 3 years, and at 7 years, DFS was $93.3 \%$. In patients with HR-positive tumors, the 7-year DFS was 94.6\%, while in HR-negative patients, the 7-year DFS was $90.7 \%$. Key secondary outcomes at 7 years included the relapse-free interval of $97.5 \%$, BC-specific survival of $98.6 \%$, and OS of $95 \% .^{70}$ This long-term data support the use of adjuvant paclitaxel and trastuzumab as a treatment option for patients with tumor size $<3 \mathrm{~cm}$ and node-negative HER2-positive BC. However, it is important to note that $50 \%$ of the patients were $<1 \mathrm{~cm}$ and another $41.2 \%$ were T-size 1 to $2 \mathrm{~cm}$. This regimen represents an important step in de-escalating therapy in early $\mathrm{BC}$ with the intent to preserve the quality of life and yet achieve excellent oncological outcomes for node-negative HER2-positive early BC. ${ }^{70}$

\section{Extended Duration of Antihuman Epidermal Growth Factor Receptor 2 Therapy}

In 2017, neratinib was approved by the Food and Drug Administration for extended adjuvant treatment of patients with early-stage HER2-positive BC after adjuvant trastuzumab-based therapy. ${ }^{71}$

So far, the overall approach has stepped up treatment by adding more targeted HER2 agents. Yet, the initiation of treatment is burdened by high cost and severe toxicity, and in some cases may be overtreatment. Therefore, it is important to update the current treatment approaches and de-escalation is a research goal to minimize adverse effects without sacrificing patient outcomes.

\section{Challenges and Future Directions}

\section{Targeting Human Epidermal Growth Factor Receptor 2/ER Crosstalk}

Although HER2 inhibition is highly effective in improving outcomes in HER2-positive BC patients, tumors expressing estrogen receptor (ER) have poorer responses to targeted therapy and are more likely to relapse. ${ }^{33,72,73}$ Current theories have revealed that in patients with HER2-positive/ER-positive tumors, a key mechanism of trastuzumab resistance could be crosstalk between HER2 and ER, most likely through PI3K pathway. It has been observed in preclinical studies with HER2-positive/ER-positive tumor models that inhibition of HER2 results in an increase in ER signaling. ${ }^{74}$

PI3K is a main component of the HER2 signaling pathway. It plays a very important role in regulating ER expression in BC. A suggested solution to the issue of HER2/ER crosstalk is to combine HER2 inhibition with ER inhibition, blocking both mechanisms. Preclinical studies have found that this strategy is effective for ER-positive tumors with PIK3CA mutations, where coadministration of PI3K inhibitors with hormone therapy increased responses. ${ }^{75}$ In a few studies, the combination of HER2 and ER inhibition improved outcomes over inhibition of either pathway alone. ${ }^{76-78}$ In the neoadjuvant setting, the simultaneous targeting of both pathways did not affect response. ${ }^{79}$

\section{Conclusion}

$\mathrm{BC}$ is a very common, complex, and heterogeneous disease. Highly malignant with poor outcomes after recurrence and metastasis, HER2-positive BC accounts for 20 to $25 \%$ of all BC. Anti-HER2 therapy is the keystone for early and advanced HER2-positive BC.

Trastuzumab has been breakthrough drug for anti-HER2 treatment. One-year treatment with trastuzumab is a standard for adjuvant therapy. Pertuzumab also showed an overall benefit in (neo) adjuvant therapy. Double-targeted adjuvant therapy can be beneficial in high-recurrence risk groups (positive lymph nodes or ER/progesterone receptor-negative patients). Extended adjuvant therapy with neratinib needs balancing therapy with adverse effects and its effects on quality of life. Treatment of residual disease after neoadjuvant therapy with T-DM1 shows promise and must be offered where possible.

Although trastuzumab, pertuzumab, lapatinib, neratinib, and T-DM1 are promising drugs, some patients may show no response or develop drug resistance after a period of treatment. There are several new anti-HER2 agents and combination studies in clinical development. With the introduction of any new treatment or regimen, care must be taken about the risks and therapeutic benefits.

\section{Financial Support and Sponsorship}

Nil.

\section{Conflicts of Interest}

There are no conflicts of interest.

\section{References}

1 Cancer Fact Sheet. Available from: https://www.who.int/ health-topicscancer\#tab=tab_1.Accessed April 1, 2021

2 Bray F, Ferlay J, Soerjomataram I, Siegel RL, Torre LA, Jemal A. Global cancer statistics 2018: GLOBOCAN estimates of incidence and mortality worldwide for 36 cancers in 185 countries. CA Cancer J Clin 2018;68(6):394-424

3 Breast Cancer. Available from: https://www.who.int/cancer/ prevention/diagnosis-screening/breast-cancer/en/. Accessed April 1, 2021

4 Malvia S, Bagadi SA, Dubey US, Saxena S. Epidemiology of breast cancer in Indian women. Asia Pac J Clin Oncol 2017;13(4):289-295

5 Mahtani R, Hineman L. Advances in HER2-positive breast cancer: novel therapies and adverse event management. J Adv Pract Oncol 2019;10(2):136-153

6 Burstein HJ. The distinctive nature of HER2-positive breast cancers. N Engl J Med 2005;353(16):1652-1654

7 Figueroa-Magalhães MC, Jelovac D, Connolly R, WolffAC.Treatment of HER2-positive breastcancer.Breast2014; 23(2):128-136

8 Baselga J, Coleman RE, Cortés J, Janni W. Advances in the management of HER2-positive early breast cancer. Crit Rev Oncol Hematol 2017;119:113-122 
9 Zurawska U, Baribeau DA, Giilck S, et al. Outcomes of her2-positive early-stage breast cancer in the trastuzumab era: a population-based study of Canadian patients. Curr Oncol 2013;20(6):e539-e545

10 Ahmed S, Sami A, Xiang J. HER2-directed therapy: current treatment options for HER2-positive breast cancer. Breast Cancer 2015;22(2):101-116

11 Pernas S, Tolaney SM. HER2-positive breast cancer: new therapeutic frontiers and overcoming resistance. Ther Adv Med Oncol 2019;11:1758835919833519

12 Pondé N, Brandão $\mathrm{M}$, El-Hachem G, Werbrouck E, Piccart M. Treatment of advanced HER2-positive breast cancer: 2018 and beyond. Cancer Treat Rev 2018;67:10-20

13 Wuerstlein R, Harbeck N. Neoadjuvant therapy for HER2positive breast cancer. Rev Recent Clin Trials 2017;12(2):81-92

14 Sharp A, Johnston SR. Dose-reduced trastuzumab emtansine: active and safe in acute hepatic dysfunction. Case Rep Oncol 2015;8(1):113-121

15 Larionov AA. Current therapies for human epidermal growth factor receptor 2-positive metastatic breast cancer patients. Front Oncol 2018;8:89

16 Lv Q, Meng Z, Yu Y, et al. Molecular mechanisms and translational therapies for human epidermal receptor 2 positive breast cancer. Int J Mol Sci 2016;17(12):2095

17 Rexer BN, Arteaga CL. Intrinsic and acquired resistance to HER2-targeted therapies in HER2 gene-amplified breast cancer: mechanisms and clinical implications. Crit Rev Oncog 2012;17(1):1-16

18 Meric-Bernstam F, Johnson AM, Dumbrava EE, et al. Advances in HER2-targeted therapy: novel agents and opportunities beyond breast and gastric cancer. Clin Cancer Res 2019;25(7):2033-2041

19 Brufsky AM. Current approaches and emerging directions in HER2-resistant breast cancer. Breast Cancer (Auckl) 2014;8:109-118

20 Baselga J. A new anti-ErbB2 strategy in the treatment of cancer: prevention of ligand-dependent ErbB2 receptor heterodimerization. Cancer Cell 2002;2(2):93-95

21 Tzahar E, Waterman H, Chen X, et al. A hierarchical network of interreceptor interactions determines signal transduction by Neu differentiation factor/neuregulin and epidermal growth factor. Mol Cell Biol 1996;16(10):5276-5287

22 Lee-Hoeflich ST, Crocker L, Yao E, et al. A central role for HER3 in HER2-amplified breast cancer: implications for targeted therapy. Cancer Res 2008;68(14):5878-5887

23 Menendez JA, Schroeder B, Peirce SK, et al. Blockade of a key region in the extracellular domain inhibits HER2 dimerization and signaling. J Natl Cancer Inst 2015;107(6):djv090

24 Franklin MC, Carey KD, Vajdos FF. Leahy DJ, de Vos AM, Sliwkowski MX. Insights into ErbB signaling from the structure of the ErbB2-pertuzumab complex. Cancer Cell 2004;5(4):317-328

25 Baselga J, Cortés J, Kim SB, et al. CLEOPATRA Study Group. Pertuzumab plus trastuzumab plus docetaxel for metastatic breast cancer. N Engl J Med 2012;366(2):109-119

26 Swain SM, Baselga J, Kim SB, et al. CLEOPATRA Study Group. Pertuzumab, trastuzumab, and docetaxel in HER2-positive metastatic breast cancer. N Engl J Med 2015;372(8):724-734

27 Baselga J, Bradbury I, Eidtmann H, et al. NeoALTTO Study Team. Lapatinib with trastuzumab for HER2-positive early breast cancer (NeoALTTO): a randomised, open-label, multicentre, phase 3 trial. Lancet 2012b;379(9816):633-640

28 Blackwell KL, Burstein HJ, Storniolo AM, et al. Overall survival benefit with lapatinib in combination with trastuzumab for patients with human epidermal growth factor receptor 2-positive metastatic breast cancer: final results from the EGF104900 Study. J Clin Oncol 2012;30(21):2585-2592
29 Bose R, Kavuri SM, Searleman AC, et al. Activating HER2 mutations in HER2 gene amplification negative breast cancer. Cancer Discov 2013;3(2):224-237

30 Rabindran SK, Discafani CM, Rosfjord EC, et al. Antitumor activity of HKI-272, an orally active, irreversible inhibitor of the HER-2 tyrosine kinase. Cancer Res 2004;64(11):3958-3965

31 Sánchez-Martín M, Pandiella A. Differential action of small molecule HER kinase inhibitors on receptor heterodimerization: therapeutic implications. Int J Cancer 2012;131(1):244-252

32 Piccart-Gebhart MJ, Procter M, Leyland-Jones B, et al. Herceptin Adjuvant (HERA) Trial Study Team. Trastuzumab after adjuvant chemotherapy in HER2-positive breast cancer. N Engl J Med 2005;353(16):1659-1672

33 Cameron D, Piccart-Gebhart MJ, Gelber RD, et al. Herceptin Adjuvant (HERA) Trial Study Team. 11 years' follow-up of trastuzumab after adjuvant chemotherapy in HER2-positive early breast cancer: final analysis of the HERceptin Adjuvant (HERA) trial. Lancet 2017;389(10075):1195-1205

34 Perez EA, Romond EH, Suman VJ, et al. Trastuzumab plus adjuvant chemotherapy for human epidermal growth factor receptor 2-positive breast cancer: planned joint analysis of overall survival from NSABP B-31 and NCCTG N9831. J Clin Oncol 2014;32(33):3744-3752

35 Gianni L, Eiermann W, Semiglazov V, et al. Neoadjuvant and adjuvant trastuzumab in patients with HER2-positive locally advanced breast cancer (NOAH): follow-up of a randomised controlled superiority trial with a parallel HER2-negative cohort. Lancet Oncol 2014;15(6):640-647

36 Goss PE, Smith IE, O'Shaughnessy J, et al. TEACH investigators. Adjuvant lapatinib for women with early-stage HER2-positive breast cancer: a randomised, controlled, phase 3 trial. Lancet Oncol 2013;14(1):88-96

37 Piccart-Gebhart M, Holmes E, Baselga J, et al. Adjuvant lapatinib and trastuzumab for early human epidermal growth factor receptor 2-positive breast cancer: Results from the randomized phase III adjuvant lapatinib and/or trastuzumab treatment optimization trial. J Clin Oncol 2016;34(10):1034-1042

38 Moreno-Aspitia A, Holmes E, Jackisch C, et al. Updated results from the phase III ALTTO trial (BIG NCCTG/Alliance N063D) comparing one year of anti-HER2 therapy with lapatinib alone $(\mathrm{L})$, trastuzumab alone $(\mathrm{T})$, their sequence $(\mathrm{T} \rightarrow \mathrm{L})$ or their combination $(\mathrm{L}+\mathrm{T})$ in the adjuvant treatment of HER2-positive early breast cance. J Clin Oncol 2017;35:2-6

39 Chan A, Delaloge S, Holmes FA, et al. ExteNET Study Group. Neratinib after trastuzumab-based adjuvant therapy in patients with HER2-positive breast cancer (ExteNET): a multicentre, randomised, double-blind, placebo-controlled, phase 3 trial. Lancet Oncol 2016;17(3):367-377

40 Martin M, Holmes FA, Ejlertsen B, et al. Neratinib after trastuzumab-based adjuvant therapy in early-stage HER2-positive breast cancer: 5-year analysis of the phase III ExteNET trial. Ann Oncol 2017;28(suppl_5) :v43-v67

41 von Minckwitz G, Procter M, de Azambuja E, et al. APHINITY Steering Committee and Investigators. Adjuvant pertuzumab and trastuzumab in early HER2-positive breast cancer. N Engl J Med 2017;377(2):122-131

42 Lambert JM, Chari RV. Ado-trastuzumab Emtansine (T-DM1): an antibody-drug conjugate (ADC) for HER2-positive breast cancer. J Med Chem 2014;57(16):6949-6964

43 von Minckwitz G, Huang CS, Mano MS, et al. KATHERINE Investigators. Trastuzumab emtansine for residual invasive HER2-positive breast cancer. N Engl J Med 2019;380(7):617-628

44 Madarnas Y, Trudeau M, Franek JA, McCready D, Pritchard KI, Messersmith H. Adjuvant/neoadjuvant trastuzumab therapy in women with HER-2/neu-overexpressing breast cancer: a systematic review. Cancer Treat Rev 2008;34(6):539-557 
45 Arnould L, Arveux P, Couturier J, et al. Pathologic complete response to trastuzumab-based neoadjuvant therapy is related to the level of HER-2 amplification. Clin Cancer Res 2007;13(21):6404-6409

46 Buzdar AU, Ibrahim NK, Francis D, et al. Significantly higher pathologic complete remission rate after neoadjuvant therapy with trastuzumab, paclitaxel, and epirubicin chemotherapy: results of a randomized trial in human epidermal growth factor receptor 2-positive operable breast cancer. J Clin Oncol 2005;23(16):3676-3685

47 Gianni L, Eiermann W, Semiglazov V, et al. Neoadjuvant chemotherapy with trastuzumab followed by adjuvant trastuzumab versus neoadjuvant chemotherapy alone, in patients with HER2-positive locally advanced breast cancer (the NOAH trial): a randomised controlled superiority trial with a parallel HER2-negative cohort. Lancet 2010;375(9712):377-384

48 Untch M, Fasching PA, Konecny GE, et al. Pathologic complete response after neoadjuvant chemotherapy plus trastuzumab predicts favorable survival in human epidermal growth factor receptor 2-overexpressing breast cancer: results from the TECHNO trial of the AGO and GBG study groups. J Clin Oncol 2011;29(25):3351-3357

49 Buzdar AU, Suman VJ, Meric-Bernstam F, et al. American College of Surgeons Oncology Group investigators. Fluorouracil, epirubicin, and cyclophosphamide (FEC-75) followed by paclitaxel plus trastuzumab versus paclitaxel plus trastuzumab followed by FEC-75 plus trastuzumab as neoadjuvant treatment for patients with HER2-positive breast cancer (Z1041): a randomised, controlled, phase 3 trial. Lancet Oncol 2013;14(13):1317-1325

50 Untch M, Rezai M, Loibl S, et al. Neoadjuvant treatment with trastuzumab in HER2-positive breast cancer: results from the GeparQuattro study. J Clin Oncol 2010;28(12):2024-2031

51 Ismael G, Hegg R, Muehlbauer S, et al. Subcutaneous versus intravenous administration of (neo)adjuvant trastuzumab in patients with HER2-positive, clinical stage I-III breast cancer (HannaH study): a phase 3, open-label, multicentre, randomised trial. Lancet Oncol 2012;13(9):869-878

52 Untch M, Loibl S, Bischoff J, et al. German Breast Group (GBG); Arbeitsgemeinschaft Gynäkologische Onkologie-Breast (AGOB) Study Group. Lapatinib versus trastuzumab in combination with neoadjuvant anthracycline-taxane-based chemotherapy (GeparQuinto, GBG 44): a randomised phase 3 trial. Lancet Oncol 2012;13(2):135-144

53 Robidoux A, Tang G, Rastogi P, et al. Lapatinib as a component of neoadjuvant therapy for HER2-positive operable breast cancer (NSABP protocol B-41): an open-label, randomised phase 3 trial. Lancet Oncol 2013;14(12):1183-1192

54 Gianni L, Pienkowski T, Im YH, et al. Efficacy and safety of neoadjuvant pertuzumab and trastuzumab in women with locally advanced, inflammatory, or early HER2-positive breast cancer (NeoSphere): a randomised multicentre, open-label, phase 2 trial. Lancet Oncol 2012;13(1):25-32

55 Gianni L, Pienkowski T, Im YH, et al. 5-year analysis of neoadjuvant pertuzumab and trastuzumab in patients with locally advanced, inflammatory, or early-stage HER2-positive breast cancer (NeoSphere): a multicentre, open-label, phase 2 randomised trial. Lancet Oncol 2016;17(6):791-800

56 Schneeweiss A, Chia S, Hickish T, et al. Pertuzumab plus trastuzumab in combination with standard neoadjuvant anthracycline-containing and anthracycline-free chemotherapy regimens in patients with HER2-positive early breast cancer: a randomized phase II cardiac safety study (TRYPHAENA) Ann Oncol 2013;24(9):2278-2284

57 Trail PA, Dubowchik GM, Lowinger TB. Antibody drug conjugates for treatment of breast cancer: Novel targets and diverse approaches in ADC design. Pharmacol Ther 2018;181:126-142
58 Verma S, Miles D, Gianni L, et al. EMILIA Study Group. Trastuzumab emtansine for HER2-positive advanced breast cancer. N Engl J Med 2012;367(19):1783-1791

59 Iwata H, Tamura K, Doi $\mathrm{T}$, et al. Trastuzumab deruxtecan (DS-8201a) in subjects with HER2-expressing solid tumors: long-term results of a large phase 1 study with multiple expansion cohorts. J Clin Oncol 2018;36:2501

60 Saura C, Thistlethwaite F, Banerji U, et al. A phase I expansion cohorts study of SYD985 in heavily pretreated patients with HER2-positive or HER2-low metastatic breast cancer. [abstract] J Clin Oncol 2018;36:1014

61 Yurkovetskiy A, Gumerov D, Ter-Ovanesyan E, et al. Nonclinical pharmacokinetics of XMT-1522, a HER2 targeting auristatin-based antibody drug conjugate [abstract]. Proc Am Assoc Cancer Res Annual Meeting 2017. Cancer Res 2017;77 13 Suppl: Abstract nr 48

62 Escrivá-de-Romaní S, Arumí M, Bellet M, Saura C. HER2positive breast cancer: current and new therapeutic strategies. Breast 2018;39:80-88

63 Esposito A, Viale G, Criscitiello C, Curigliano G. A clinical perspective on escalating or de-escalating adjuvant therapy in HER2+ breast cancer. Expert Rev Clin Pharmacol 2019;12(1):9-16

64 Joensuu H, Bono P, Kataja V, et al. Fluorouracil, epirubicin, and cyclophosphamide with either docetaxel or vinorelbine, with or without trastuzumab, as adjuvant treatments of breast cancer: final results of the FinHer Trial. J Clin Oncol 2009;27(34):5685-5692

65 Slamon D, Eiermann W, Robert N, et al. Breast Cancer International Research Group. Adjuvant trastuzumab in HER2positive breast cancer. N Engl J Med 2011;365(14):1273-1283

66 Guarneri V, Frassoldati A, Bruzzi P, et al. Multicentric, randomized phase III trial of two different adjuvant chemotherapy regimens plus three versus twelve months of trastuzumab in patients with HER2- positive breast cancer (Short-HER Trial; NCT00629278) Clin Breast Cancer 2008;8(5):453-456

67 Conte PF, Bisagni G, Frassoldati A, et al. 9 weeks vs. 1 year adjuvant trastuzumab in combination with chemotherapy: results of the phase III multicentric Italian study Short-HER. [abstract] J Clin Oncol 2017;35:501

68 Earl HM, Cameron DA, Miles D, et al. The PERSEPHONE trial: duration of trastuzumab with chemotherapy in women with HER-2 positive early breast cancer. [abstract] J Clin Oncol 2012;30:660

69 Earl HM, Hiller L, Vallier AL, et al. PERSEPHONE Steering Committee and Trial Investigators. 6 versus 12 months of adjuvant trastuzumab for HER2-positive early breast cancer (PERSEPHONE): 4-year disease-free survival results of a randomised phase 3 non-inferiority trial. Lancet 2019;393(10191) :2599-2612

70 Tolaney SM, Guo H, Pernas S, et al. Seven-year follow-up analysis of adjuvant paclitaxel and trastuzumab trial for node-negative, human epidermal growth factor receptor 2-positive breast cancer. J Clin Oncol 2019;37(22):1868-1875

71 U.S. Food \& Drugs Administration, Prescribing Information for Nerlynx. Available from: http://www.accessdata.fda.gov/drugsatfda_docs/label/2017/208051s000lbl.pdf. Accessed April 10, 2021.

72 André F, O’Regan R, Ozguroglu M, et al. Everolimus for women with trastuzumab-resistant, HER2-positive, advanced breast cancer (BOLERO-3): a randomised, double-blind, placebo-controlled phase 3 trial. Lancet Oncol 2014;15(6):580-591

73 De Laurentiis M, Arpino G, Massarelli E, et al. A meta-analysis on the interaction between HER-2 expression and response to endocrine treatment in advanced breast cancer. Clin Cancer Res 2005;11(13):4741-4748

74 Xia W, Bacus S, Hegde P, et al. A model of acquired autoresistance to a potent ErbB2 tyrosine kinase inhibitor and a 
therapeutic strategy to prevent its onset in breast cancer. Proc Natl Acad Sci U S A 2006;103(20):7795-7800

75 Bosch A, Li Z, Bergamaschi A, et al. PI3K inhibition results in enhanced estrogen receptor function and dependence in hormone receptor-positive breast cancer. Sci Transl Med 2015;7(283):283ra51

76 Johnston S, Pippen J Jr, Pivot X, et al. Lapatinib combined with letrozole versus letrozole and placebo as first-line therapy for postmenopausal hormone receptor-positive metastatic breast cancer. J Clin Oncol 2009;27(33):5538-5546

77 Kaufman B, Mackey JR, Clemens MR, et al. Trastuzumab plus anastrozole versus anastrozole alone for the treatment of postmenopausal women with human epidermal growth factor receptor 2-positive, hormone receptor-positive metastatic breast cancer: results from the randomized phase III TAnDEM study. J Clin Oncol 2009;27(33):5529-5537

78 Rimawi MF, Mayer IA, Forero A, et al. Multicenter phase II study of neoadjuvant lapatinib and trastuzumab with hormonal therapy and without chemotherapy in patients with human epidermal growth factor receptor 2-overexpressing breast cancer: TBCRC 006. J Clin Oncol 2013;31(14):1726-1731

79 Nitz UA, Gluz O, Christgen M, et al. West-German Study Group (WSG)-ADAPT Investigators. De-escalation strategies in HER2-positive early breast cancer (EBC): Final analysis of the WSG-ADAPT HER2+/HR- phases II trial: efficacy, safety, and predictive markers for 12 weeks of neoadjuvant dual blockade with trastuzumab and pertuzumab \pm weekly paclitaxel. Ann Oncol 2017;28:2768-2772 